\title{
Estado nutricional e práticas de educação nutricional em escolares ${ }^{\#}$
}

\author{
Nutritional status and nutritional education practices in school
}

\author{
Luciane Peter Grillo* \\ Milenna Larissa Conceição** \\ Cristina Henschel de Matos* \\ Leo Lynce Valle de Lacerda*
}

\begin{abstract}
Resumo
A situação nutricional da população infantil de um país é essencial para aferir a evolução das condições de saúde e de vida da população em geral. Estudo transversal de natureza quantitativa que teve como objetivo avaliar o perfil antropométrico de 400 usuários do Programa Nacional de Alimentação Escolar da rede municipal de ensino de Canoinhas, Santa Catarina e desenvolver atividades de educação nutricional com a comunidade escolar. Foram avaliados o peso, a altura e a circunferência da cintura. O diagnóstico nutricional antropométrico foi por meio do índice de massa corporal por idade, com padrões de referências da Organização Mundial de Saúde, os pontos de corte do Ministério da Saúde e também por meio da circunferência da cintura. A prevalência de escolares com excesso de peso e obesidade central foi de $23 \%$. A segunda etapa da pesquisa foi composta por cinco oficinas de nutrição, abordando o tema da alimentação saudável na infância, sendo o conteúdo adaptado conforme o público participante da comunidade escolar. Os resultados encontrados confirmam o período de transição nutricional que o país vem enfrentando. Além de servir como alerta para os programas de saúde pública que trabalham com a alimentação escolar, pois com o elevado número de escolares acima do peso deve-se repensar as estratégias de educação alimentar que utilizam a escola como local formador de hábitos alimentares mais saudáveis.
\end{abstract}

Palavras-chave: Educação Alimentar e Nutricional. Antropometria. Criança. Avaliação Nutricional.

\begin{abstract}
The nutritional status of the child population of a country is essential to measure the evolution of the health and life of the general population. Cross-quantitative study that aimed to evaluate the anthropometric profile of 400 users of the National School Feeding Programed municipal in Canoinhas, Santa Catarina and develop nutrition education activities with the school community. They evaluated the weight, height and waist circumference. The anthropometric nutritional diagnosis was through the body mass index for age, with reference standard of the World Health Organization and the cutoff points of the Ministry of Health and also by waist circumference. The prevalence of children with overweight and central obesity was $23 \%$. The second stage of the research consisted of five workshops in nutrition, addressing the theme of healthy eating in childhood, with content adapted as the participating public school community. The results confirm the nutritional transition period in the country, in addition to use as a warning to the public health programs that work with school feeding, because with the high number of school overweight should rethink nutrition education strategies use the school as a trainer place of healthier eating habits.
\end{abstract}

Keywords: Food and Nutrition Education. Anthropometry. Child. Nutrition Assessment.

DOI: $10.15343 / 0104-7809.20164002230238$

\footnotetext{
\# Este artigo originou-se da dissertação intitulada: "Promoção da alimentação saudável: proposta de implantação de um programa de educação nutricional em escolas", de Milenna Larissa Conceição. Apresentado em 2010 ao Programa de Mestrado Profissional em Saúde e Gestão do Trabalho da Universidade do Vale do Itajaí (UNIVALI).

* Universidade do Vale do Itajaí (UNIVALI). Itajaí - SC, Brasil. E-mail: grillo@univali.br

** Secretaria de Educação do Município de Canoinhas, SC, Brasil.

Os autores declaram não haver conflitos de interesse.
} 


\section{INTRODUÇÃO}

O período de vida entre seis e 10 anos de idade se caracteriza por maior atividade física e ritmo de crescimento constante com ganho mais acentuado de peso próximo ao estirão da adolescência. Há crescente independência da criança que, na maioria das vezes, inicia as atividades escolares por volta dos sete anos e forma novos laços sociais com adultos e indivíduos da mesma idade. Essas transformações, aliadas ao processo educacional, são determinantes para o aprendizado em todas as áreas e para o estabelecimento de novos hábitos ${ }^{1}$.

A alimentação adequada da criança e do adolescente garante qualidade na vida adulta. Crianças e adolescentes têm o direito de receber a alimentação escolar oferecida gratuitamente nas escolas públicas e garantida pelo Programa Nacional de Alimentação Escolar (PNAE). O PNAE prevê a transferência, às escolas públicas, de recursos financeiros destinados à aquisição de gêneros alimentícios para o preparo da alimentação escolar oferecida aos alunos matriculados na educação infantil (em creches e em pré-escolas), no ensino fundamental e médio das escolas da rede pública que constam no censo escolar realizado pelo Ministério da Educação no ano anterior ao atendimento ${ }^{2}$.

Durante as últimas décadas observou-se alteração no perfil nutricional das crianças brasileiras, com a redução da desnutrição e aumento do sobrepeso e da obesidade. Entre os fatores apontados como expressivos para explicar essa situação estão a expansão da cobertura de saneamento e a ampliação do acesso aos serviços básicos de saúde ${ }^{3}$. Além disso, evidências indicam que o meio ambiente, permeado pelas condições materiais de vida e pelo acesso à educação, determina padrões característicos de saúde e doença na criança. Variáveis como renda familiar e escolaridade estão condicionadas, em última instância, à forma de inserção das famílias no processo de produção, refletindo na aquisição de alimentos $\mathrm{e}$, consequentemente, no estado nutricional ${ }^{4}$.

O último estudo realizado no Brasil para investigar o estado nutricional de crianças encontrou $34,8 \%$ das crianças com idade entre cinco e nove anos com diagnóstico de excesso de peso, indicando que $16,6 \%$ deste público já são considerados obesos ${ }^{5}$. Estudo global publicado recentemente sobre a prevalência de sobrepeso e obesidade durante o período de 1980-2013 observou um aumento significativo nos países em desenvolvimento, de 8,1\% em 1980 para 12,9\% em 2013 para os meninos e de $8,4 \%$ a $13,4 \%$ em meninas, respectivamente. Em 2013, nos países desenvolvidos, estes valores foram de $23,9 \%$ em meninos e $22,6 \%$ em meninas 6 .

As crianças constituem, portanto, um dos principais grupos-alvo para estratégias de prevenção e controle dos distúrbios nutricionais, não só devido às suas características como grupo de risco, mas também por conta das possibilidades de sucesso das ações a serem implementadas. Nessa perspectiva, o ambiente escolar se torna bastante atrativo para investigar a ocorrência dos distúrbios nutricionais entre as crianças, considerando a abrangência, geralmente elevada, do sistema escolar entre os alunos ingressantes no ensino fundamental e o apoio da rede escolar para a obtenção de informações sobre a criança e sua família, com vistas ao desenvolvimento de programas de prevenção de alterações nutricionais. Ainda dentro deste contexto, a Educação Alimentar e Nutricional é vista como estratégia para a promoção de hábitos alimentares saudáveis, considerando-se que a escola oferece um espaço apropriado para o desenvolvimento destas ações ${ }^{7}$.

Este estudo teve como objetivos identificar o estado nutricional por meio da antropometria (peso, altura, circunferência da cintura) dos usuários do PNAE matriculados do $1^{\circ}$ ao $4^{\circ}$ ano do ensino fundamental e desenvolver atividades de educação nutricional com a comunidade escolar.

\section{MÉTODOS}

Esta pesquisa, de caráter transversal, foi desenvolvida na rede municipal de ensino da cidade de Canoinhas, no estado de Santa Catarina, sul do Brasil.

O município de Canoinhas está situado no planalto norte de Santa Catarina. Apresenta uma área de $1.143,5 \mathrm{Km}^{2}$ e possui 52.765 habitantes, sendo que aproximadamente $75 \%$ 
da população residem na zona urbana e 25\% se concentram na zona rural ${ }^{8}$.

Conforme o levantamento realizado pela Secretaria de Educação do município de Canoinhas (2009) todas as escolas municipais estavam vinculadas ao PNAE. O número de alunos matriculados do $1^{\circ}$ ao $4^{\circ}$ ano do ensino fundamental na rede municipal totalizava 2.569 nas 21 escolas (dez delas localizadas na zona urbana e onze na zona rural). Por questões operacionais, as escolas rurais foram excluídas da pesquisa.

A população deste estudo foi composta por escolares de seis a dez anos, cursando do $1^{\circ}$ ao $4^{\circ}$ ano do ensino fundamental, matriculados nas escolas urbanas no ano de 2010. O número de alunos totalizou 1.608 escolares (887 do sexo masculino e 721 do sexo feminino). Os avaliados foram selecionados por meio de amostra casual simples proporcional ao sexo com erro máximo de 5\% e uma confiança de $95 \%$. Sob estes parâmetros a amostra foi composta por 400 indivíduos, sendo 221 do sexo masculino e 179 do sexo feminino.

O presente estudo foi aprovado pela Comissão de Ética em Pesquisa da Universidade do Vale do Itajaí (CAAE- 0940.0.000.22310). Os pais ou responsáveis dos alunos que fizeram parte da pesquisa assinaram o termo de consentimento livre esclarecido, no qual constavam todas as etapas do estudo.

A coleta dos dados antropométricos ocorreu nos meses de maio a julho de 2010 e foi realizada por profissionais habilitados e treinados para esse trabalho. A idade dos avaliados foi calculada conforme a data de nascimento, obtida a partir do último censo escolar disponibilizado pela Secretaria de Educação.

A avaliação antropométrica foi realizada mediante coleta das seguintes variáveis: peso, altura e circunferência da cintura. Após a coleta do peso $(\mathrm{kg})$ e altura $(\mathrm{m})$, calculou-se o Índice de Massa Corporal (IMC). Utilizaram-se, para classificação, o padrão de referência da Organização Mundial da Saúde ${ }^{9}$ e os pontos de corte do Ministério da Saúde ${ }^{10}$.

Para a medida da circunferência da cintura, considerou-se a parte mais estreita do tronco. Para a classificação da circunferência da cintura como indicador isolado de risco cardiovascular, utilizaram-se os pontos de corte propostos por Taylor et al. ${ }^{11}$.

As práticas de educação alimentar e nutricional foram baseadas na concepção de Paulo Freire que engloba o diálogo e a comunicação gerando críticas e problematização $^{12}$, sendo implementadas em duas das cinco oficinas de nutrição, que ocorreram em datas diferentes, com duração média de 60 minutos cada, abrangendo públicos diferentes em cada uma delas. Todas tiveram como tema central a alimentação saudável na infância. As oficinas ocorreram no segundo semestre de 2010 e foram desenvolvidas na escola e na comunidade envolvendo o maior número de alunos matriculados do $1^{\circ}$ ao $4^{\circ}$ ano da rede municipal de ensino, bem como pais, professores e funcionários das escolas.

Na primeira oficina de nutrição participaram o corpo docente e funcionários da escola e, na segunda, pais ou responsáveis pelos escolares. Os preceitos de Paulo Freire ${ }^{12}$ nortearam esta primeira etapa, na qual foram apresentados os resultados da pesquisa e abordadas as situações comuns que são encontradas na rotina da alimentação das crianças. Discutiuse com o grupo o contexto das situações vivenciadas, os problemas e angústias vividas e neste momento, estimulou-se a colaboração de todos os integrantes para a construção coletiva de ações que pudessem melhorar os hábitos alimentares das crianças, bem como auxiliar nas dificuldades enfrentadas pelos professores e funcionários. Todo o processo foi mediado pelo diálogo, sendo estimulada a reflexão das situações problema e discutidas e estabelecidas as responsabilidades de cada uma das partes.

A terceira oficina, intitulada "Momento saudável", foi desenvolvida com os escolares matriculados do $1^{\circ}$ ao $4^{\circ}$ ano. Nessa etapa, utilizaram-se materiais lúdicos para explicar a maneira saudável de se alimentar, incluindo a pirâmide alimentar com réplicas de alimentos, teatros com fantoches e a figura do palhaço chamado "Sr. Saúde", que sugeria alterações para melhorar a dieta e ter um bom comportamento à mesa. Ao final do encontro, os participantes receberam figuras ilustrativas, com tema relacionado à alimentação saudável para colorir. Todas as atividades foram desenvolvidas com os participantes 
divididos por grupos, conforme o ano escolar, adaptando-se as práticas educativas conforme a necessidade e a faixa etária.

Na quarta oficina, desenvolveu-se a "Semana da Criança Saudável", durante a qual foi servido aos escolares um café da manhã completo, composto por cereais integrais, frutas e laticínios, seguido por um passeio ciclístico no bairro onde se localiza a escola.

A última atividade, nomeada "Sabor e saúde", consistiu em uma oficina culinária onde foram desenvolvidas preparações saudáveis na cozinha experimental de uma escola técnica localizada no mesmo bairro da escola. As receitas para essa oficina contemplavam a inclusão, na dieta dos escolares, de alimentos com boa qualidade nutricional, como frutas, verduras, cereais integrais e carnes magras. Tais preparações precisavam ser de baixo

\section{RESULTADOS}

Foram avaliados os escolares matriculados nas dez escolas urbanas do município. A amostra avaliada foi composta por 400 escolares, sendo $55 \%(n=221)$ do sexo masculino e $45 \%(n=179)$ do sexo feminino.

O diagnóstico nutricional pode ser observado na Tabela 1. Verifica-se a predominância da normalidade $(76 \%, \mathrm{n}=304)$, seguida do excesso de peso $(23 \%, n=92)$, com diferenças não significativas em relação ao sexo, a exceção da normalidade que apresentou percentual no sexo feminino $(80,4 \%)$ significativamente custo, saborosas e de fácil preparo para que pudessem ser inseridas no hábito alimentar da família. Foram preparadas receitas de alto valor nutritivo, como suco de frutas com vegetais, panquecas com a inclusão do vegetal na massa e da fruta no recheio, sanduíches integrais recheados com hortaliças e carnes magras. A avaliação das atividades educativas foi realizada a partir de um questionário autoaplicado no qual os sujeitos expressavam o seu grau de satisfação com as atividades realizadas.

Os dados foram processados e analisados por meio do aplicativo Excel e Statistica versão 10. A análise descritiva foi feita a partir de distribuição de frequência e medidas de tendência central e dispersão. $\mathrm{Na}$ comparação entre proporções foi utilizado o teste para duas proporções com nível de significância de 5\%.

( $p=0,0394)$ maior que no masculino $(72,4 \%)$. Apesar da tendência de maior percentual de obesidade no sexo masculino $(12,2 \%)$ em relação ao feminino $(7,3 \%)$, a diferença não foi significativa $(p=0,4976)$.

O estudo aferiu a circunferência da cintura para avaliar o risco cardiovascular do público pesquisado.

Os resultados mostram que $23 \%$ dos avaliados apresentaram acúmulo de gordura na região do tronco (Tabela 2) sem diferenças significativas entre os sexos.

Tabela 1- Perfil nutricional dos escolares: número absoluto (n) e percentual (\%) de acordo com o diagnóstico nutricional, Canoinhas, SC, 2010.

\begin{tabular}{|c|c|c|c|c|c|c|c|}
\hline \multirow{3}{*}{$\begin{array}{l}\text { Diagnóstico } \\
\text { nutricional }\end{array}$} & \multicolumn{4}{|c|}{ Sexo } & \multirow[t]{3}{*}{ Valor de $\mathbf{p}^{*}$} & \multirow{2}{*}{\multicolumn{2}{|c|}{ Total }} \\
\hline & \multicolumn{2}{|c|}{ Masculino } & \multicolumn{2}{|c|}{ Feminino } & & & \\
\hline & $n$ & $\%$ & $\mathrm{n}$ & $\%$ & & $\mathrm{n}$ & $\%$ \\
\hline Magreza & 3 & 1,4 & 1 & 0,6 & 0,7594 & 4 & 1,0 \\
\hline Normalidade & 160 & 72,4 & 144 & 80,4 & 0,0394 & 304 & 76,0 \\
\hline Sobrepeso & 31 & 14,0 & 21 & 11,7 & 0,8092 & 52 & 13,0 \\
\hline Obesidade & 27 & 12,2 & 13 & 7,3 & 0,4976 & 40 & 10,0 \\
\hline Total & 221 & 100,0 & 179 & 100,0 & - & 400 & 100,0 \\
\hline
\end{tabular}

*no teste entre duas proporções ao nível de 5\%. 
Tabela 2- Número absoluto e percentual de acúmulo de gordura na região do tronco nos escolares, Canoinhas, SC, 2010.

\begin{tabular}{|c|c|c|c|c|c|c|c|}
\hline \multirow{3}{*}{$\begin{array}{l}\text { Riscos } \\
\text { cardiovasculares }\end{array}$} & \multicolumn{4}{|c|}{ Sexo } & \multirow[t]{3}{*}{ Valor de $\mathrm{p}^{*}$} & \multirow{2}{*}{\multicolumn{2}{|c|}{ Total }} \\
\hline & \multicolumn{2}{|c|}{ Masculino } & \multicolumn{2}{|c|}{ Feminino } & & & \\
\hline & $n$ & $\%$ & $\mathrm{n}$ & $\%$ & & $\mathrm{n}$ & $\%$ \\
\hline Risco & 46 & 20,8 & 45 & 25 & 0,6256 & 91 & 22,0 \\
\hline Normalidade & 175 & 79,2 & 134 & 74,9 & 0,3363 & 309 & 78,0 \\
\hline Total & 221 & 100,0 & 179 & 100,0 & - & 400 & 100,0 \\
\hline
\end{tabular}

*no teste entre duas proporções ao nível de 5\%.

O Gráfico 1 apresenta dados referentes observa-se valores menores de sobrepeso e ao diagnóstico nutricional de escolares obesidade nos escolares deste estudo para participantes deste estudo (município de ambos os sexos, comparativamente aos dados Canoinhas), da região sul do Brasil $^{5}$, no qual regionais e nacionais.

Gráfico 1- Comparativo do diagnóstico nutricional de escolares de Canoinhas, Região Sul e Brasil.

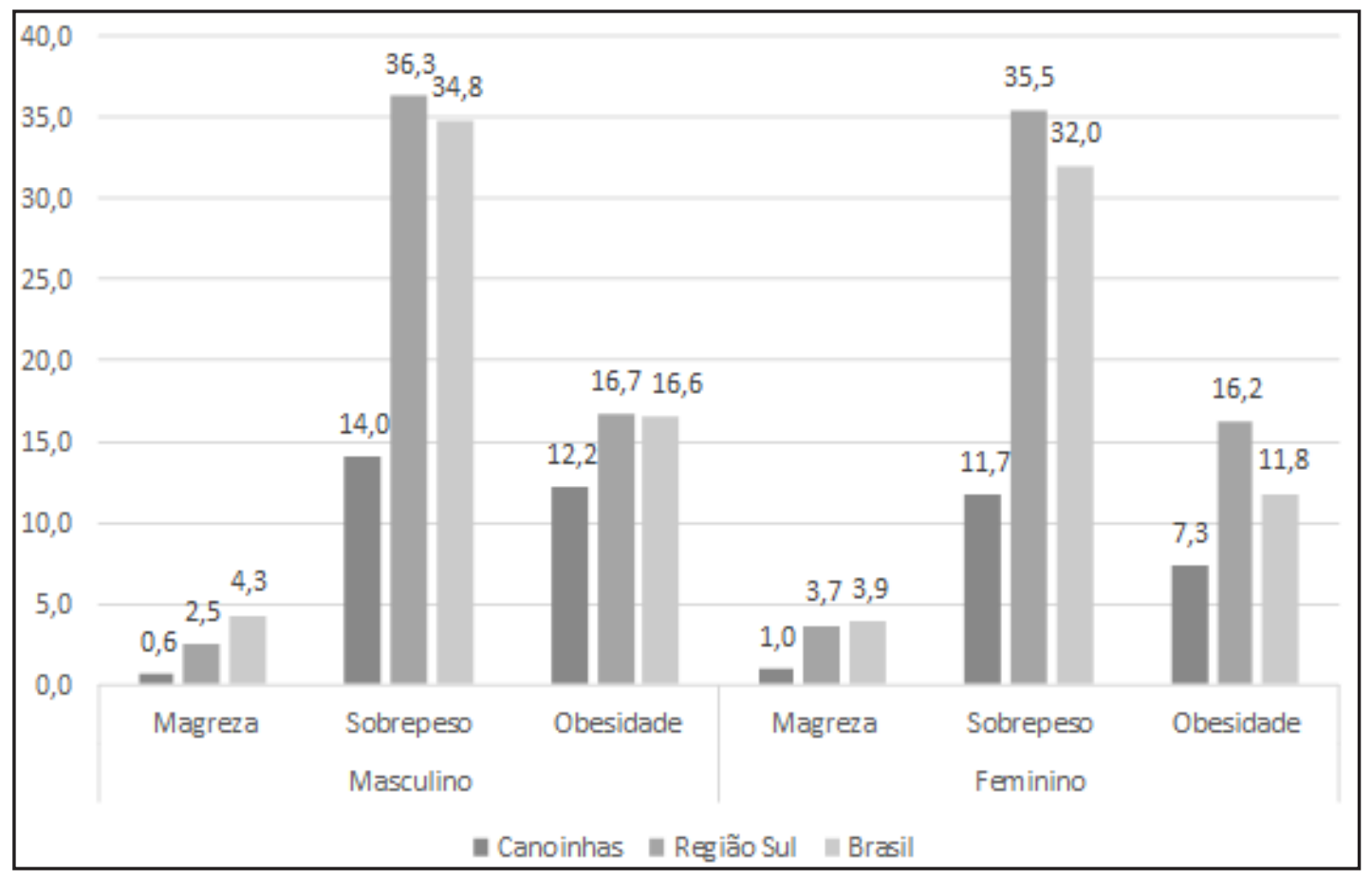

Fonte: Canoinhas: dados da pesquisa. Região Sul e Brasil: IBGE, 2010.

Ao final do período em que foram realizadas as oficinas de nutrição, solicitou-se aos pais ou responsáveis pelos escolares e aos funcionários da escola o preenchimento da escala hedônica para avaliar o grau de satisfação do público com as atividades desenvolvidas (Tabela 3). 
Tabela 3- Distribuição (\%) do grau de satisfação dos participantes das oficinas de nutrição, Canoinhas, SC, 2010.

\begin{tabular}{lcc}
\hline \multicolumn{1}{c}{ Grau de Satisfação } & $\mathbf{n}$ & \% \\
\hline Desgostei extremamente & - & 0,0 \\
Desgostei moderadamente & - & 0,0 \\
Nem gostei/nem desgostei & - & 0,0 \\
Gostei moderadamente & 3 & 1,0 \\
Gostei extremamente & 97 & 97,0 \\
\hline
\end{tabular}

\section{DISCUSSÃO}

O estudo evidenciou alta prevalência de excesso de peso $(23 \% ; n=92)$ e baixa prevalência de magreza $(1 \% ; n=4)$. O sexo masculino totalizou $26,2 \%(n=58)$ de excesso de peso, sendo $12,2 \% \quad(n=27)$ diagnosticados com obesidade. No sexo feminino foi possível observar o excesso de peso em $11,7 \% \quad(n=21)$ das avaliadas, sendo $7,3 \%(n=13)$ das meninas consideradas obesas. Esses resultados estão abaixo dos encontrados na região sul do Brasil e no país, respectivamente, tanto para o sexo masculino $(16,7 \% ; 16,6 \%)$ quanto para o sexo feminino $(16,2 \% ; 11,8 \%)^{5}$.

No sudeste brasileiro, no estado de São Paulo, 680 escolares de Sorocaba foram avaliados, $22,1 \%$ estavam com excesso de peso ${ }^{13}$. Em Minas Gerais (Uberaba), pesquisadores avaliaram 458 escolares com idade entre seis e 10 anos e encontraram a prevalência de sobrepeso e obesidade em 15,5\% e 14\%, respectivamente ${ }^{14}$. Em Niterói (RJ), autores pesquisaram o estado nutricional em 328 escolares e encontraram $25,7 \%$ de excesso de peso ${ }^{15}$. Estes autores sugerem que a detecção precoce de crianças com estado nutricional inadequado, associada a ações voltadas à promoção da saúde, com intervenções nas escolas, nas famílias e com o acesso de todos a informações sobre os determinantes e as consequências da obesidade poderão resultar em um maior controle de distúrbios nutricionais nesse grupo etário ${ }^{14}$.

No nordeste do país, em Alagoas, pesquisa envolvendo 860 crianças encontrou $24 \%$ dos participantes com excesso de peso ${ }^{16}$. Na Bahia, numa amostra de 224 escolares entre 6 e 9 anos de idade, a prevalência de sobrepeso foi de $7,6 \%$ e 5,8 de obesidade ${ }^{17}$. Em Campina Grande (Paraíba) encontrou-se 23,5\% de excesso de peso, sendo mais frequente entre os alunos da rede privada ${ }^{18}$.

No norte brasileiro foi investigado o estado nutricional de escolares de seis a 10 anos da rede municipal da sede de Mucajaí, Roraima, Belém. Esse estudo encontrou 46,29\% de baixo peso e $10,24 \%$ de excesso de peso, permitindo concluir que estes escolares, diferente do que acontece no restante do país, não estão em processo de transição nutricional ${ }^{19}$.

Outros estudos realizados na região sul do Brasil também verificaram a prevalência do excesso de peso em crianças. Na capital de Santa Catarina, Florianópolis, escolares com idade entre sete e 10 anos foram avaliados e os autores identificaram a prevalência de sobrepeso e obesidade mais elevada em meninos $(36,2 \%)$ do que em meninas $(32,7 \%)^{20}$. Em quatro municípios do Rio Grande do Sul (Pelotas, Chapada, Santa Cruz do Sul e um do Vale do Taquari) foram encontradas prevalências superiores ao presente estudo, 37,7\%, 30\%, $28,6 \%$ e $40,4 \%$, respectivamente ${ }^{21-24}$. No interior do Paraná (Maringá) foram avaliados 4609 escolares com idade entre seis e 11 anos e a prevalência de excesso de peso foi de $24,5 \%{ }^{25}$.

Quanto à adiposidade central aferida, conforme a medida da circunferência da cintura foi possível observar que 23\% (n=92) da amostra estudada neste estudo apresentou risco cardiovascular. A literatura ainda é restrita neste parâmetro para determinar a obesidade central na infância ${ }^{26}$. O resultado deste estudo foi inferior aos de Pelotas ${ }^{21}$ (24\%) e do município do Vale do Taquari $^{24}(29,8 \%)$ e superior aos de Sorocaba ${ }^{13}$ e Santa Cruz do Sul ${ }^{23}$ onde 15,4\% e $19,1 \%$ dos avaliados apresentaram adiposidade central.

Esses resultados, com exceção do estudo desenvolvido no norte do Brasil, evidenciam que a transição nutricional vem ocorrendo de modo semelhante em todo o país e que independe do sexo e condição socioeconômica. É indiscutível que o excesso de peso traz prejuízos à saúde dos indivíduos. 
Diante dos percentuais encontrados neste estudo e nas demais pesquisas realizadas nas diversas regiões do Brasil foram planejadas ações de prevenção ao excesso de peso e sugeridas medidas auxiliares no combate à obesidade, utilizando a escola como espaço adequado para o desenvolvimento de hábitos alimentares saudáveis. Assim, iniciaram-se as atividades de educação nutricional. Em todas as oficinas foram apresentados os resultados da pesquisa no município e fornecidas orientações para uma alimentação saudável na infância.

É fato incontestável a importância da alimentação saudável, completa, variada e agradável ao paladar para a promoção da saúde, sobretudo para organismos jovens, em fase de desenvolvimento, tanto para a prevenção quanto para o controle de doenças crônicas não transmissíveis, cuja prevalência vem aumentando significativamente ${ }^{27-30}$.

A Educação Alimentar e Nutricional ultrapassa os processos de comunicação e de informação, ficando evidente que a forma com que esta é desenvolvida influencia de maneira decisiva nos resultados. Ressalta-se que para a comunicação ser efetiva, deve ser pautada no reconhecimento dos diferentes saberes e práticas, formação de vínculo entre os sujeitos que integram o processo, construção partilhada de saberes, práticas e soluções, relações horizontais e busca de soluções contextualizadas ${ }^{31}$.

Dentro deste contexto, é provável que a abordagem de situações cotidianas relacionada à alimentação das crianças, bem como o levantamento das dificuldades e sugestões para melhorar os hábitos alimentares em casa (trabalhados na primeira oficina), juntamente com a participação direta ou indireta de todos os sujeitos (funcionários, professores, alunos, pais e comunidade) envolvidos no processo e a metodologia lúdica utilizada foram os responsáveis por tornar a comunicação entre educador e educando mais efetiva, resultando nos altos percentuais de satisfação com as oficinas (97\%). Destaca-se a importância do desenvolvimento de ações educativas que visem à promoção da autonomia dos sujeitos baseada na interdisciplinaridade, respeitando as culturas, valorizando a história e a diversidade regional e ao mesmo tempo em que reconheçam os saberes populares e fomentem a biodiversidade local $^{32,33}$, como consequência melhora o conhecimento sobre nutrição, as atitudes e o comportamento alimentar, podendo também influenciar os hábitos alimentares da família e comunidade.

\section{CONCLUSÃO}

Com este estudo foi possível constatar que o excesso de peso associado à obesidade abdominal atinge praticamente $1 / 4$ dos escolares usuários do PNAE no município de Canoinhas, sul do Brasil.

Sugere-se que seja abordada nas escolas da rede pública de ensino, de forma multidisciplinar, a promoção de uma alimentação saudável com vistas à prevenção das consequências do excesso de peso na saúde dos indivíduos, principalmente na faixa etária estudada, entre seis e 10 anos, considerando que eles ainda não chegaram à adolescência, fase em que os hábitos alimentares podem sofrer maior influência do meio em que vivem, das novas amizades, da mídia, enfim, do ambiente no qual estão inseridos.

Outra questão que vale ressaltar é a importância do acompanhamento nutricional por profissional habilitado nos casos em que foram diagnosticados excesso de peso e adiposidade central, visando orientações nutricionais para reverter o quadro já instalado e prevenir consequências do excesso de peso na vida adulta.

Acredita-se que a metodologia utilizada nas primeiras oficinas, na qual se valorizou as experiências vividas, o diálogo e a problematização das situações enfrentadas, tanto pelos professores e funcionários como pelos pais, facilitou a implementação das outras etapas, resultando no alto percentual de satisfação das atividades educativas, melhorando a comunicação entre as partes e como consequência influenciou positivamente nos hábitos alimentares dos envolvidos.

Sugere-se também a continuação deste estudo, abrangendo os alunos matriculados nas escolas localizadas no meio rural, bem como os 
adolescentes que estejam cursando do $5^{\circ}$ até o 9a ano do ensino fundamental. Entende-se que, quanto mais cedo forem identificados os casos de distúrbios nutricionais, melhores resultados serão obtidos no processo de educação e/ou acompanhamento nutricional e maiores serão as chances de adequação do estado nutricional, evitando o aparecimento de problemas de saúde relacionados ao excesso de peso, tais como as doenças crônicas não transmissíveis, e colaborando para a redução dos gastos públicos com o tratamento dessas doenças.

\section{REFERÊNCIAS}

1. Sociedade Brasileira de Pediatria. Avaliação nutricional da criança e do adolescente - Manual de orientação. São Paulo: Sociedade Brasileira de Pediatria, 2009.64p.

2. Brasil. Ministério da Educação. Resolução FNDE /CD № 038, de 23 de agosto de 2004. Programa Nacional de Alimentação Escolar PNAE. Brasilia, DF, 2004. Disponível em: https://www.fnde.gov.br/fndelegis/action/UrlPublicasAction.php?acao=abrirAtoPublico\&sgl_ tipo=RES\&num_ato=00000038\&seq_ato=000\&vlr_ano=2004\&sgl_orgao=CD/FNDE/MEC. Acesso em: 11 mai. 2016.

3. Victora CG. et al. Saúde de mães e crianças no Brasil: progressos e desafios. Lancet (serie). 2011; 32-46.

4. Schmidt MI. et al. Doenças crônicas não transmissíveis no Brasil: carga e desafios atuais. Lancet, 2011; 377:1949-61.

5. Instituto Brasileiro de Geografia e Estatística. Pesquisa Orçamentos Familiares 2008-2009: antropometria e estado nutricional de crianças, adolescentes e adultos no Brasil. Rio de Janeiro:IBGE, 2010a.150p.

6. NG M. et al. Global, regional, and national prevalence of overweight and obesity in children and adults during 1980-2013: a systematic analysis for the Global Burden of Disease Study 2013. Lancet (online). 2014; 384: 766-81.

7. Ramos FP, DA Silva Santos LA, Reis ABC. Educação alimentar e nutricional em escolares: uma revisão de literatura. Cad. Saúde Pública. 2013; 29(11): 2147-61.

8. Instituto Brasileiro de Geografia e Estatística, Censo Populacional 2010, 2010b. Disponível em: http://www.cidades.ibge.gov.br/ xtras/perfil.php?lang=\&codmun=420380\&search=santa-catarina $\mid$ canoinhas. Acesso em 11 mai. 2016.

9. Who. Child Growth Standards. Disponível em: http://www.who.int/childgrowth/standards/en/ . Acesso em: 10 nov. 2010.

10. Brasil. Ministério da Saúde. Protocolos do Sistema de Vigilância Alimentar e Nutricional - SISVAN na assistência à saúde. Brasilia: MS, 2008. 61p.

11. Taylor RW. et al. Evaluation of waist circumference, waist-to-hip ratio, and the conicity index as screening tools for high trunk fat mass, as measured by dual-energy X-ray absorptiometry, in children aged 3-19 y. Am. J. Clin. Nut. 2000; 72(2): 490-95.

12. Freire P. Educação como prática de liberdade. 34 ed. Rio de Janeiro: Paz e Terra; 2011.

13. Mazaro IAR. et al. Obesidade e fatores de risco cardiovascular em estudantes de Sorocaba, SP. Rev. assoc. med. bras. 2011; 57(6): 674-680.

14. Brito JBSM, Walsh IAP, Damião R. Estado nutricional de escolares de uma escola pública estadual. REFACS (online). 2013; 1(1): $17-23$.

15. Vasconcelos MB, Anjos LA, Vasconcellos MTL. Estado nutricional e tempo de tela de escolares da Rede Pública de Ensino Fundamental de Niterói, Rio de Janeiro, Brasil. Cad. saúde públ. 2013; 29(4): 713-722.

16. Ramires EKNM. et al. Estado nutricional de crianças e adolescentes de um município do semiárido do Nordeste brasileiro. Rev. paul pediatr. 2014; 32(3): 200-207.

17. Carvalho IL. et al. Estado nutricional da rede pública de ensino. Rev. Rene. 2014; 15(2): 291-297.

18. Medeiros CCM. et al. Estado nutricional e hábitos de vida em escolares. Rev. bras. cresc. desenv. hum. 2011; 21(3): $789-797$.

19. Galiasso CAF. et al. O perfil nutricional de escolares de 6 a 10 anos da rede municipal da sede de Mucajaí - Roraima - Brasil. Nucleus. 2014; 11(2): 113-124.

20. Bernardo CO. et al. Fatores associados ao estado nutricional de escolares de 7 a 10 anos: aspectos sociodemográficos, de consumo alimentar e estado nutricional dos pais. Rev. bras. epidemiol. 2012; 15(3): 651-661.

21. Kaufmann CC, Albernaz EP. et al. Prevalência e fatores associados ao excesso de peso em crianças de uma coorte no Sul do Brasil. Rev. ciên \& saúde. 2013; 6 (3): 172-180.

22. Polla SF, Scherer F. Perfil alimentar e nutricional de escolares da rede municipal de ensino de um município do interior do Rio Grande do Sul. Cad. Saúde colet. 2011; 19(1): 111-116.

23. Tornquist L. et al. Excesso de peso e pressão arterial elevada em escolares: prevalência e fatores associados. Jour. hum. growth and develop. 2015; 25(2): 216-223.

24. Piletti D, Strack MH, Adami FS. Risco cardiovascular de crianças e adolescentes. Cader. Pedag. 2015; 12(1): 9-21.

25. Rosaneli CF et al. Aumento da pressão arterial e obesidade na infância: Uma avaliação transversal de 4609 escolares. Arq. bras. cardiol. [online]. 2014; PP.0-0.

26. Magalhães EIS et al. Perímetro da cintura, relação cintura/estatura e perímetro do pescoço como parâmetros na avaliação da obesidade central em crianças. Rev. paul. pediatr. 2014; 32(3): 273-282.

27. Brasil. Ministério da Saúde. Política Nacional de Alimentação e Nutrição. Brasília: MS, 2012a. 88p.

28. Yokota RTC. et al. Projeto "a escola promovendo hábitos alimentares saudáveis": comparação de duas estratégias de educação nutricional no Distrito Federal, Brasil. Rev. Nutr. 2010; 23(1): 37-47.

29. Andrade J. et al. Intervenções escolares para redução da obesidade infantil: uma revisão sistemática. 2015; Ciên. \& saúde 8(2): 72-78.

30. Bernart A, Zanardo VPS. Educação nutricional para crianças em escolas públicas de Erechim/RS. 2011; Vivências 7(13): 71-79.

31. Brasil. Ministério do Desenvolvimento Social e Combate a Fome. Marco de referência de educação alimentar e nutricional para políticas públicas. - Brasilia: MDS, 2012 b. 
32. Da Silva Santos, LA. O fazer educação alimentar e nutricional: algumas contribuições para reflexão. Ciênc. saúde coletiva. 2012; 17(2): 453-62.

33. Brasil. Ministério da Educação. Cartilha Nacional da Alimentação Escolar. Brasília: ME, 2015. 88p. 